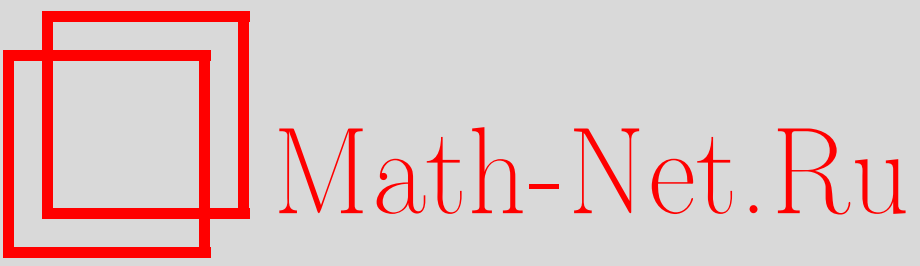

В. Х. Хаханян, Независимость частного случая аксиомы выбора в интуиционистской теории множеств, УМН, 1997, том 52, выпуск 4, 219-220

DOI: https://doi.org/10.4213/rm878

Использование Общероссийского математического портала Math-Net.Ru подразумевает, что вы прочитали и согласны с пользовательским соглашением

http://www.mathnet.ru/rus/agreement

Параметры загрузки:

IP: 3.80 .181 .102

26 апреля 2023 г., 17:21:27 


\title{
НЕЗАВИСИМОСТЬ ЧАСТНОГО СЛУЧАЯ АКСИОМЫ ВЫБОРА В ИНТУИЦИОНИСТСКОЙ ТЕОРИИ МНОЖЕСТВ
}

\author{
В. Х. ХАХАНЯН
}

В настоящей заметке доказывается независимость частного случая аксиомы выбора в интуиционистской двусортной теории множеств. Аналогичное доказательство может быть проведено и для односортной теории множеств.

1. Язык теории $Z F I 2$. Язьк теории включает: два сорта переменных (по натуральным числам $(m, n, p, k, \ldots)$ и по множествам $(x, y, z, t, \ldots))$, символы для предикатов принадлежности натурального числа множеству: $\epsilon^{0}$, множества - множеству: $\epsilon^{1}$, и равенства для натуральных чисел, числовой константы 0 , логических связок и кванторов по переменным любого сорта и всех примитивно-рекурсивных функций. Далее верхние индексы у предикатов принадлежности опускаем, так как из текста всегда ясно, какой из предикатов имеется ввиду.

2. Аксиомы теории $Z F I 2$. Это все аксиомы арифметики, включая аксиомы логики предикатов для двух сортов переменных и принцип индукции по всем формулам языка, которые строятся из атомарных, как обычно. Вторую группу составляют теоретико-множественные аксиомы пары, суммы, степени, бесконечности, трансфинитной индукции по множествам, выделения, подстановки и экстенсиональности, записанные для двусортного язька. Например, аксиомы выделения и подстановки имеют такой вид:

$$
\begin{aligned}
& \forall z \exists y[\forall m(m \in y \leftrightarrow m \in z \wedge \varphi(m)) \wedge \forall t(t \in y \leftrightarrow t \in z \wedge \psi(t))], \\
& \forall x\{[\forall m \in x \exists ! y \varphi(m, y) \wedge \forall t \in x \exists ! y \psi(t, y)] \\
&\rightarrow \exists z[\forall m \in x \downarrow \exists y \in z \varphi(m, y) \wedge \forall t \in x \exists y \in z \psi(t, y)]\} .
\end{aligned}
$$

Здесь на $\varphi$ и $\psi$ накладываются обычные ограничения и в них входят параметры любого сорта.

3. Рассматриваемый случай аксиомы выбора имеет вид:

$$
\begin{aligned}
A C^{0} & \rightleftharpoons \forall n \exists m \varphi(n, m) \\
& \rightarrow \exists x[\operatorname{Fn}(x) \wedge \operatorname{Dom}(x)=\omega \wedge \operatorname{Rng}(x) \subseteq \omega \wedge \forall n \exists m(\varphi(n, m) \wedge\langle n, m\rangle \in x)] .
\end{aligned}
$$

Здесь $\operatorname{Fn}(x)$ - формула, утверждающая, что множество $x$ - функция, $\omega$ - множество натуральных чисел. Аксиома утверждает возможность выбора из всех сущих $m$ для $n$ одного.

Отметим следующие обстоятельства.

1) Если ограничить посылку $A C^{0}$ требованием единственности для существования $\forall n \exists ! m$ $\varphi(n, m)$, то такая аксиома $A C^{0}$ ! вьводима в $Z F I 2$.

Teopema 1. $Z F I 2 \vdash\{\forall n \exists ! m \varphi(n, m) \rightarrow \exists x[\operatorname{Fn}(x) \wedge \operatorname{Dom}(x)=\omega \wedge \operatorname{Rng}(x) \subseteq \omega \wedge$ $\forall n \exists m(\varphi(n, m) \wedge\langle n, m\rangle \in x)]\}$.

ДокАЗАТЕЛЬСТво. Применяем аксиомы подстановки и выделения.

2) Если добавить к $Z F I 2$ аксиому "собирания" (которая отличается от аксиомы подстановки отсутствием требования единственности в посылке) и усилить таким образом $Z F I 2$, см. [3], то аналогичное доказательство для вывода $A C^{0}$ все же не проходит; мы покажем, что доказательства нет.

3) В заключении аксиомы подстановки можно требовать, чтобы существовало не множество, а функция с нужным свойством: это не изменит силы аксиомы; аналогичная замена в аксиоме "собирания" даст неприемлемую интуиционистски аксиому (обозначим ее $A C F$ ), так как тогда выводится стандартная форма аксиомы выбора $A C$, а также полный закон исключенного третьего. 
TeOpema 2. B ZFI2 выводимо:

a) $A C \leftrightarrow A C F$, где $A C \rightleftharpoons \forall x \in B \exists y \in x \rightarrow \exists f[\operatorname{Fn}(f) \wedge \operatorname{Dom}(f)=B \wedge \forall x \in B$ $f(x) \in x]-$ стандартная форма аксиомы выбора;

б) $A C F \rightarrow \psi \vee \neg \psi$ для любой $\psi$ языка $Z F I 2$.

Доказательство б) дали в 1975 г. N. D. Goodman и F. Myhill. Приведем здесь краткое доказательство Г. Е. Минца из [4]. Пусть $B=\{a, b\}$, где $a=\varnothing$ и $b=\{x \mid x=0 \wedge \psi\}, \mathbb{C}=\{0,1\}$, $\varphi(x, y) \rightleftharpoons(x=a \wedge y=0) \vee(x=b \wedge y=1)$ и $\psi$ - фиксированная формула. Используя аксиомш пары, экстенсиональности и факт, что $0 \neq 1$, получим $a=b \vee a \neq b$ и затем $\psi \vee \neg \psi$.

4. Рассмотрим теперь $A C^{0}$. Установить ее совместимость с $Z F I 2$ можно даже интуиционистски и даже с $Z F I 2+C T$, где $C T$ - тезис Чёрча и имеет вид $\forall n \exists m \varphi(n, m) \rightarrow \exists e \forall n$ $\varphi(n,\{e\}(n))$; здесь $e$ - гёделев номер общерекурсивной функции и $\{e\}(n)$ есть значение функции с номером $e$ на аргументе $n$. Тезис Чёрча утверждает, что все всюду определенные функции натурального аргумента являются вычислимыми.

TEOpema 3. $Z F I 2+C T \vdash A C^{0}$.

ДокАзАТЕЛЬСТво. Применяем тезис Чёрча и аксиому выделения.

Обозначим теорию $Z F I 2+$ аксиома "собирания” через $Z F C 2$.

Теорема 4. Теория $Z F C 2+A C^{0}$ непротиворечива относительно теории $Z F C 2$.

ДокАЗАТЕЛЬСтво. Применяем теорему 3 и основную теорему из [1].

TEOPEMA 5. $Z F C 2 \not A C^{0}$.

ДоКАЗАТЕЛЬСтво. Предположим $Z F C 2 \vdash A C^{0}$. Тогда $Z F C 2+C T$ ! $\vdash A C^{0}$ и $Z F C 2+$ $C T$ ! $\vdash C T$. Последнее доказываем так: пусть $\forall n \exists m \varphi(n, m)$; применяя $A C^{0}$, имеем $\exists f \forall n$ $\varphi(n, f(n))$, т.е. $\forall n \exists ! m(\varphi(n, m) \wedge f(n)=m)$; применяем $C T$ ! (отличающийся от $C T$ требованием единственности существования в посылке) и получаем заключение для $C T$. Однако следствие из [2] утверждает, что $Z F C 2+C T$ ! $\forall C T$. Теперь получаем, $Z F C 2+C T ! \forall A C^{0}$ и $Z F C 2+A C^{0} ! \forall A C^{0}$ и $Z F C 2 \forall \forall A C^{0}$.

\section{СПИСОК ЛИТЕРАТУРЫ}

[1] Хаханян В. Х. // Вестник МГУ. Сер. матем., мех. 1980. № 5. С. 3-7. [2] Хаханян В. Х. // Докл. АН СCCP. 1980. Т. 252. № 5. С. 1070-1074. [3] Friedman H., Ščedrov A. // Adv. Math. 1985. № 57. Р. 1-13. [4] РЖ “Математика”, 1979. № 5. 5А59. 